\title{
Construcción de la cultura de calidad en un hospital público a partir de la gestión humana: tensiones y paradojas"
}

\author{
Construction of quality culture in a public hospital from a \\ human management perspective: tensions and paradoxes
}

Recibido: marzo 14 de 2010 | Revisado: agosto 7 de 2010 | Aceptado: noviembre 8 de 2010

\author{
Claudia María García Álvarez ** \\ SANDRA MILENA RODRÍGUEZ LÓPEZ \\ Karin Viviana SuÁrez Puentes \\ Universidad Piloto de Colombia, Bogotá
}

RESUMEN

El objetivo de este estudio fue indagar acerca del significado del discurso de calidad en un hospital público de Bogotá y, particularmente, el papel que juega la gestión humana en la construcción de la cultura de calidad, con el fin de vislumbrar los intereses a los que responde este discurso y las tensiones que suscita. En el proceso de construcción una cultura de calidad se observa que existe una concepción ambigua del trabajador: como eje fundamental en la construcción del sistema de calidad y como costo que debe ser minimizado. Se concluye que el discurso de calidad responde a una lógica económica-administrativa, cuyas medidas y categorías legitiman un modelo de salud basado en el mercado y con esto se evidencia el papel de la gestión humana -a través del uso de técnicas psicológicas- en la reproducción de este modelo.

Palabras clave autores

Calidad en salud, gestión de la calidad, gestión humana, cultura organizacional.

Palabras clave descriptores

Psicología organizacional, análisis de discurso, investigación cualitativa.

Para citar este artículo. García A. C. M., Rodrig L. S. M. \& Suárez P. K. V. (2011). Construcción de la cultura de calidad en un hospital público a partir de la gestión humana: tensiones y paradojas. Universitas Psychologica, 10 (3), 841-853.

* Este artículo es el resultado del proyecto de investigación titulado Significado de la calidad en la gestión y los servicios prestados por los hospitales adscritos a la secretaría de salud de Bogotá, liderado por Claudia García miembro del grupo de investigación GOCE (Gestión y Organizaciones desde Corrientes Emergentes). En este proyecto participaron como investigadores auxiliares los estudiantes de último año, Sandra Milena Rodríguez, Lina Puentes, Karin Viviana Suárez y Víctor Pérez.

** Universidad Piloto de Colombia. Carrera 9 No. 45 A 44 Piso 2 Tel. (57-1) 3322900 Extensión: 325. Bogotá DC, Colombia. Correos electrónicos: clamagar@ yahoo.es, celloka@hotmail.com, goretica_lulu@ hotmail.com

\section{A B S T R A C T}

The purpose of this study was to explore about the meaning of quality discourse in a public hospital in Bogota, and particularly the role that human management plays in the construction of a quality culture, in order to glimpse the interests to which this discourse responds, and the tensions aroused. In the process of quality culture construction, it is observed an ambiguous conception of worker: as a fundamental element in the construction of quality system and as a cost to be minimized. It is concluded that the quality discourse responds to an economic-administrative logic which measurements and categories legitimates a health model based on the market and besides it is exposed the role of Human Resource Management -through the use of psychological techniques- in the reproduction of this model.

Health quality, quality management, human resource management, organizational culture.

Organizational psychology, discourse analysis, qualitative research. Key words authors

Key words plus 


\section{Psicología, cultura organizacional y gestión humana}

Este trabajo se desarrolla en el marco de la Psicología crítica, y busca comprender cómo en las organizaciones, particularmente las organizaciones del sector salud, se han venido incorporando técnicas y conocimientos psicológicos, dentro de las prácticas de regulación y administración del comportamiento de las personas (Rose, 1998). En ese sentido, las áreas de gestión humana se convierten en escenarios donde las tecnologías psicológicas son implementadas para producir ciertos resultados en términos de la conducta humana. Según Rose, la Psicología no es solo una técnica de individualización, sino que es una técnica de espacios y relaciones que se funde con todas aquellas prácticas que están a disposición de autoridades administradoras de colectividades humanas. Así, la gerencia de recursos humanos ${ }^{1}$ de las empresas, a través del uso de saberes psicológicos, ha asumido el papel de alinear la fuerza laboral con los objetivos corporativos (Legge, 1995), y en este marco, la cultura organizacional se ha convertido no solo en una herramienta conceptual para explicar el funcionamiento de la organización como un sistema psicosocial, sino en "un conjunto de saberes y técnicas que posibilitan intervenir e influir en este sistema de manera que pueda ser alineado con la estrategia corporativa" (García, 2009, p. 278). En otras palabras, la cultura organizacional se convierte en una tecnología social que la Psicología ha contribuido a ensamblar ${ }^{2}$, orientada a la administración de lo colectivo ${ }^{3}$, en

1 Se ha denominado esta área de diversas formas: gerencia de recursos humanos, área de gestión humana, talento humano, desarrollo humano. Cada denominación con una connotación diferente pero con un objetivo común: la administración de las personas en las organizaciones para el logro de fines específicos.

2 El concepto de cultura es el resultado de la contribución de diferentes disciplinas, particularmente la Antropología; la Psicología a través de autores como Edgar Schein, también ha aportado en su construcción, sobre todo en el marco de las organizaciones.

3 La administración o gestión cultural se puede llevar a cabo a través de diversas técnicas basadas en saberes psicológicos tales como la capacitación -producción y propagación de ciertos significados-, la administración de incentivos para reforzar ciertos comportamientos y disminuir otros, la selección de sujetos afines con los valores corporativos, la evaluación del desempeño con base en criterios específicos, etc. este caso, el comportamiento organizacional. Rose (1999) señala que la psicología es un conjunto de técnicas -saberes y prácticas- que contribuyen a la construcción de sujetos de acuerdo con objetivos específicos -reforma, cura, eficiencia- en respuesta a un orden social particular, y en el caso de las empresas, "(...) industrial success was to be based upon the utilization of expert technologies for the management of subjectivity" (p. 118).

Entre otras cosas, en este estudio se busca mostrar cómo la psicología en el marco de la gestión humana, contribuye a la producción de cierto orden social a través del uso de técnicas orientadas a la producción de sujetos que reproducen y legitiman dicho orden, el cual no necesariamente contribuye al bienestar y calidad de vida del trabajador.

Este trabajo hace parte de una serie de estudios que se han llevado a cabo desde el año 2005 en el seno de diferentes hospitales públicos de Bogotá (García, 2007; García, 2008; Valdés, 2008; AbadíaBarrero et al., 2008; Abadía-Barrero \& Oviedo, 2009), que han mostrado el predominio de una lógica racional y económica-administrativa en los hospitales, y con esto, el deterioro de las condiciones laborales de los trabajadores de la salud ${ }^{4}$ e incremento de las dificultades y obstáculos que enfrentan los pacientes para acceder a un servicio de salud. Particularmente se busca analizar en medio de estas transformaciones derivadas del actual modelo de salud implementado en Colombia, el papel de la gestión humana en la administración de los cambios llevados a cabo en los hospitales públicos, específicamente el cambio hacia una cultura de calidad.

4 Este deterioro de las condiciones laborales en el contexto contemporáneo no es exclusivo del sector de la salud, como lo demuestran múltiples estudios realizados en diferentes sectores y en diversos países que dan cuenta del incremento de la inseguridad y precariedad laboral (Sennett, 2000; Castells, 1998; Gorz, 1997; Urrea, 1999; Escobar, 2005) y de las iniciativas empresariales para la captura de la subjetividad (Gorz, 1997; De la Garza, 2002; Escobar, 2005). 


\section{La calidad en el marco del Sistema de Seguridad Social en Salud en Colombia}

En el año 1993 se llevó a cabo el proceso de transformación del Sistema de Seguridad Social en Salud en Colombia, en consonancia con las reformas de los sistemas de salud de otros países, frente a la crisis de los Estados de bienestar (Goretty, Abadía \& Martínez, 2008). Este proceso se orienta hacia la adopción de un modelo en el que los servicios de salud se incorporan al mercado, buscando la eliminación de la financiación del Estado, desde el supuesto de que la regulación de la salud a partir del mercado, lleva a la eficiencia en la prestación del servicio en tanto los prestadores, para ser elegidos en medio de múltiples ofertas, deben autorregularse. En este marco, se establece la calidad como uno de los principios más importantes, frente a las demandas y exigencias del cliente.

De esta manera, el Ministerio de la Protección Social en la perspectiva de implementar procesos técnicos eficientes para una prestación de servicios en términos de calidad, cantidad y oportunidad, se propuso desarrollar el Sistema de Gestión de Calidad bajo los parámetros de la Norma Técnica de Calidad para la Gestión Pública NTCGP-1000:2004, constituyéndose en una herramienta de gestión y mejoramiento, para lograr un servicio eficiente y oportuno (Ministerio de la Protección Social, 2008).

En el Sistema de Garantía de Calidad se incorporan cuatro componentes: el sistema único de acreditación de IPS (Instituciones Prestadoras de Servicio de Salud) y de EPS (Entidades Promotoras de Salud); el sistema de información a los usuarios, y EPS; la auditoria para el mejoramiento de la calidad, y el sistema único de habilitación de prestadores y EPS (Kerguelén, 2003).

A nivel local estas políticas de calidad se han venido reforzando desde la Secretaría de Salud de Bogotá, en el marco de las políticas del Gobierno distrital, a través del desarrollo de estrategias para mejorar los servicios de salud que ofrecen las instituciones que conforman la red pública de Empresas Sociales del Estado del Distrito Capital. En este sentido, en el año 2000, la Secretaría creó el
Premio a la Gestión y Calidad de los Servicios de Salud. Los objetivos de este han sido el reconocimiento del esfuerzo de las instituciones en el logro del mejoramiento de la gestión, y la prestación a las instituciones de una herramienta de autoevaluación, que les permita promover mecanismos para optimizar la calidad de la atención (Luengas, 2003).

En el marco del hospital público, el proceso de implementación del Sistema de Gestión de Calidad ha implicado el desarrollo de un conjunto de acciones sistemáticas, continuas y deliberadas, dirigidas a evitar, prevenir o resolver oportunamente situaciones que puedan afectar negativamente la obtención de los mayores beneficios posibles para los pacientes, con los menores riesgos (Kerguelén, 2003). La gestión de la calidad se concibe como un proceso integral y, en ese orden de ideas, toda la organización debe focalizar los esfuerzos hacia el propósito de lograr resultados de calidad. Sin embargo, son los trabajadores quienes, en últimas, aterrizan la filosofía de calidad a través de sus acciones y, por eso, el área de gestión humana tiene un papel fundamental en la gestión de una cultura orientada a la calidad, que lleve a alinear los comportamientos de los trabajadores a este propósito organizacional.

No obstante, en la definición de calidad y específicamente, calidad en salud, entran en juego diversos centros de poder como organizaciones de profesionales, asignadores de recursos y entidades estatales, que tratarán de imponer un paradigma específico en correspondencia con sus intereses particulares (Kerguelén, 2003). Desde la perspectiva de este trabajo, la pregunta acerca del significado de calidad, parte del supuesto de que los discursos que circulan en el contexto de las organizaciones, y particularmente el discurso de calidad, son construcciones sociales y, por tanto, resultado de un proceso de lucha de significados, en el que ciertas formas de interpretar la realidad, en este caso, lo que significa calidad en el contexto de la salud, termina imponiéndose y paulatinamente naturalizándose, en respuesta a determinados intereses y en perjuicio de otros (Mumby, 1997). 
El objetivo de este trabajo fue indagar acerca del significado del discurso de calidad en un hospital público de Bogotá, en el marco del modelo de salud y, particularmente, el papel que juega la gestión humana en la construcción de la cultura de calidad, con el fin de vislumbrar los intereses a los que responde este discurso y las tensiones que suscita.

\section{Método}

Para dar respuesta a este objetivo, se realizó un estudio de caso en un hospital público de Bogotá de tercer nivel, que ha sido certificado y que además ha obtenido reconocimientos por la implementación exitosa del sistema de gestión de calidad ${ }^{5}$. Se realizaron entrevistas a profundidad a trabajadores del área asistencial (médicos, enfermeras, camilleros), trabajadores del área de Gestión Humana (Gerente de Talento Humano y Coordinador de Bienestar) y al Coordinador de Calidad. Una vez transcritas las entrevistas, se identificaron categorías en cada una de ellas y se triangularon los datos a través de matrices de sentido (Bonilla \& Rodríguez, 1997). Los resultados que se describen y analizan a continuación, ubicados en tres categorías, dan cuenta del proceso de implementación de la cultura de la calidad en el hospital y del papel de la gestión humana en el mismo, y de las paradojas y tensiones que suscita el discurso de calidad.

\section{Resultados}

\section{Gestión de la calidad: camino hacia la sostenibilidad financiera}

Con la transformación de los hospitales públicos en Empresas Sociales del Estado se inicia un proceso de implementación de reformas que llevan a

5 Aunque se trata de un estudio de caso, este hace parte de un conjunto de investigaciones desarrolladas en varios hospitales públicos de Bogotá (García, 2006; García, 2007; Valdés, 2008; Abadía-Barrero et al., 2008), que dan cuenta de los cambios implementados en los hospitales, en su proceso de transformación a Empresas Sociales del Estado; cambios que validan lo hallado en este estudio y que a su vez se derivan de la implementación de políticas basadas en el modelo de salud adoptado en Colombia. generar nuevos controles orientados a garantizar la sostenibilidad financiera de los hospitales. Esta exigencia con respecto a la gestión financiera y administrativa, está en conexión con la obligación de adoptar las normas relacionadas con el Sistema de Garantía de Calidad:

El hospital hace ya va para once años se transformó en una Empresa Social del Estado; antes era una institución pública vinculada a la Secretaría de Salud, como un apéndice de la Secretaría de Salud, (...) con esa transformación vino la implementación de alguna normatividad relacionada con la calidad y con el control interno que era la ley 87 del 93 de control interno que ya se venía implementando y el decreto 2174 del 96 que era el decreto del sistema obligatorio de garantía de la calidad. Entonces, con esa transformación, el hospital inició con unos procesos propios de gestión como hacía ya la parte financiera y las cuentas; ese era el punto crítico, porque de un momento a otro se tenía que hacer el cambio de comenzar a actuar como empresa, a facturar, a generar sus propios ingresos. (Coordinador de Calidad)

La calidad, entonces, tiene que ver, en un primer momento, con el manejo eficiente de recursos y la generación de ingresos, es decir, con el funcionamiento del hospital como empresa.

El hospital, a partir del impulso inicial y con base en las herramientas del sistema de control interno que había empezado a implementar, comienza a generar una cultura de la documentación a través del registro de información, que permitiera hacer visible las acciones realizadas en los diferentes procesos y poder así controlar y asegurar que todos los procesos se ajustaran a los criterios establecidos:

Dentro de ese proceso se recurrió a que las herramientas de sistema control interno se fueran masificando en el hospital, con base al procedimiento de todos los procesos que existen en el hospital, tener esos manuales; entonces, de ahí comenzó como esa cultura de la documentación del seguimiento de tareas, de algunos comités operativos que controlaran algunas gestiones. (Coordinador de Calidad) 
El logro de la calidad significa, entonces, el establecimiento de estándares y protocolos - manuales de procesos y procedimientos- que conduzcan a la reducción de la variabilidad de los procesos y, en ese sentido, la cultura de la documentación va conectada con una cultura de la evaluación y seguimiento, que lleve a reducir la brecha entre lo esperado y lo ejecutado.

Con la decisión de la implementación de la norma ISO 9002, el hospital consolida el proceso, dándole fuerza al enfoque de gestión hacia la calidad, que implica la construcción de una cultura de documentación, control y seguimiento, y mejoramiento y prevención. Esto, según el Coordinador de Calidad, es la base para la implementación de un sistema de gestión de la calidad.

Llegando como al año 2000, se tomó la decisión de que el hospital se certificara y, entonces el hospital optó por implementar la norma ISO 9002, en el área de cirugía y, pues, ahí ya comenzó ese proceso de la documentación de manuales, de la implementación de controles al proceso, del seguimiento a través de acciones correctivas, de darle un enfoque más de gestión hacia la calidad, frente a lo que se estaba realizando; entonces, después de realizar ese proceso ya quedaron las bases de todo.

Todo este proceso ha tenido sus frutos, de acuerdo con los funcionarios, por los premios y reconocimientos que ha obtenido el hospital, los cuales permiten sustentar la imagen de un hospital que hace las cosas bien y, por supuesto, esto lo lleva a hacerse más atractivo ante el cliente potencial que contribuirá a incrementar los ingresos.

Empieza, pues, la lluvia de reconocimientos a la institución, de premios externos, de certificaciones, de visitas de otras partes, de convertirse el hospital en el hospital modelo (...). Todo esto hace que el hospital termine siendo un hospital financieramente muy sólido. (Médica)

Los premios, además de contribuir a la construcción de una imagen atractiva a la luz del cliente, son refuerzos importantes para la construcción de una cultura de calidad dentro del hospital, en la medida en que terminan legitimando entre los trabajadores el proceso que se viene implementando. Sin embargo, es sobre todo a través de la Gestión Humana que la dirección desarrolla estrategias, para difundir la filosofía de calidad y construir una cultura sólida en torno a la calidad.

\section{Gestión Humana y cultura de calidad}

La implementación del proceso de calidad a través de la introducción de estándares, controles y protocolos, ya de por sí implica un cambio en la cultura. Pero más importante aún es que los trabajadores le encuentren sentido a ese proceso e incorporen la filosofía de calidad en su rutina diaria; en un contexto laboral en el que se promueve un trabajador autónomo (García \& Carvajal, 2007) se busca que las acciones del trabajador no estén coaccionadas, sino que se den por la propia iniciativa de este, y al mismo tiempo y paradójicamente, que estén alineadas con los objetivos corporativos. En palabras de Rose (1998), en un contexto donde se promueve el emprendimiento y autonomía del trabajador/ciudadano, se gobierna a través de la libertad y deseos de estos, definiendo lo que es deseable y bueno a partir de la autoridad de expertos. Esto se traduce en la empresa, en un proceso de gestión cultural, que busca que los trabajadores, al identificarse con la organización, piensen y actúen en consonancia con esta (Peters \& Waterman, 1984; Schein, 1985). Así, Gestión Humana se encarga de gestionar el proceso de cambio hacia una cultura de la calidad, a través de diferentes estrategias que buscan que el trabajador in-corpore el discurso de calidad.

El hospital tiene 20 programas que están direccionados por el área de calidad; cada departamento es responsable de un programa, Talento Humano como tal es responsable de la Escuela de Calidad (...). Nosotros difundimos esa parte para que la gente la interiorice y se sienta partícipe del asunto (...). Esta estrategia de difusión está orientada al proceso de acreditación, proceso para habilitación, o sea nosotros llevamos eso colgado todos los días. (Gerente de Talento Humano) 
El departamento de Talento Humano a través de la gestión cultural busca construir un lenguaje unificado en torno a la filosofía de calidad, que permita alinear al trabajador con todo el sistema de gestión de calidad. Esto implica que para que se concreticen las políticas de calidad, los trabajadores deben incorporar la filosofía que sustenta dichas políticas, de manera que el discurso de la calidad tenga sentido y oriente las acciones.

Otra de las estrategias de gestión humana que busca vincular el trabajador a la cultura de la organización, es el programa de incentivos, el cual tiene como fin resaltar diversos aspectos en los trabajadores tales como el buen desempeño individual o colectivo, el logro de las metas establecidas por cada área, reforzando así comportamientos afines a la cultura deseada.

Hay un plan de incentivos en el hospital, que puede ser por grupos, individuales, donde a la gente se le reconoce de alguna manera, si se ha destacado (...); está el plan de reconocimiento para el mejor empleado por la actitud de servicio, el mejor empleado por entrega a su trabajo, (...) pero con que a ti te entreguen un reconocimiento, una medalla, un desayuno, un almuerzo, cualquier cosa de esas, la gente se siente motivada con eso. (Gerente de Talento Humano)

Por otro lado, la formación hace parte de este conjunto de estrategias para promover la cultura de calidad, que lleva a los trabajadores a considerar al hospital como una escuela de aprendizaje.

Aquí a ti te enseñan, aquí te involucran, aquí te dicen cómo debes hacer las cosas y después, cuando ya te sientes ducho, te sueltan; entonces en ese ejercicio, el hospital es lo que te digo: una muy buena escuela, una escuela que procura que su gente se quede, que su gente se sienta motivada, que se capacite. (Gerente de Gestión Humana)

Precisamente, casualmente hace ocho días recibí un diplomado, un grado sobre eso, a nosotros nos están haciendo varios cursos, cursos de calidad, epidemiología, cursos sobre relaciones humanas, todos esos cursitos nos los están haciendo ahora en la actualidad, y eso es muy bueno, no solamente para practicarlo allí, sino para practicar en cualquier sitio. (Enfermera)

Se observa entonces que un eje central en la implementación del sistema de calidad es el diseño de estrategias de difusión de significados que se despliegan a través de múltiples programas, buscando construir al trabajador deseado. El programa de inducción se constituye en otra estrategia fundamental para materializar el discurso de calidad.

La inducción es el proceso donde uno apalanca todo; el explicarle a la gente a qué viene a este hospital y cómo este está diseñando todos sus procesos (...). (Coordinador de Calidad)

En estos procesos de formación, el trabajador adquiere nuevos conocimientos y herramientas que lo hacen más competente y, por lo tanto, más atractivo para otras entidades que buscan este tipo de perfiles. Entonces, paradójicamente, la inversión que hace el hospital en el trabajador para integrarlo y retenerlo, se convierte en una oportunidad para el empleado de conseguir mejores opciones laborales.

Porque si en algo podemos llevarnos el orgullo, es que el hospital $\mathrm{X}$ es escuela y es centro de formación. Aquí no nos guardamos nada y, obviamente, el entrenamiento que tú adquieres aquí es muy específico y especializado, entonces eso hace que tú puedas competir en otros espacios y que haya gente que simplemente de las otras empresas nos los quiten. (Gerente de Talento Humano)

A pesar de la inversión que hace el hospital para integrar a los trabajadores, la Gerente de Talento Humano reconoce el incremento del índice de rotación.

El índice de rotación del hospital se ha disparado un poquito en los dos últimos años; según la literatura debe ser del $2.9 \%$ no más, nosotros estamos en un $3.8 \%$ del índice de rotación. 
Entre las razones para explicar estos niveles de rotación, se encuentra las formas de contratación que ha adoptado el hospital, a partir de su transformación hacia una empresa sostenible financieramente.

En la segunda administración se consolidan los sistemas de calidad y se empieza el proceso de certificación ISO, y se busca como el mejor equilibrio entre la contratación de planta y la contratación de prestación de servicios. (Médica)

Es así como todo este proceso de gestión de la calidad derivó en el aumento de formas de contratación flexible que favorecieran la autosostenibilidad del hospital, pero que, paradójicamente, van en detrimento de la estabilidad de los trabajadores.

En ese sentido, es importante resaltar que en el hospital, la mayor parte de los trabajadores están vinculados por medio de cooperativas ${ }^{6}$-el total de trabajadores es de mil trescientos y por cooperativa se tienen aproximadamente 900 personas, entre administrativos y asistenciales-. Esta diferencia en la contratación se refleja en las desiguales condiciones laborales entre los trabajadores de planta y los cooperados.

El único beneficio que tenemos en el hospital, es saber que tenemos un trabajo, pero beneficios como tal no tenemos ninguno; si vemos, no tenemos ningunas prestaciones, como yo se lo comentaba anteriormente, a nosotros con el salario nos descuentan (...), no tenemos ninguna bonificación siquiera, si queremos sobrepasar un poquito eso nos toca hacer turnos entre sí, y pagárnoslos; eso cuando se lo permiten a uno. (Enfermera vinculada por cooperativa)

En las condiciones del trabajador es un poco más difícil, porque empezando por las diferencias que

6 A partir de marzo de 2009 el Alcalde Mayor de Bogotá, Samuel Moreno Rojas con base en estudios realizados por la Secretaría Distrital de Salud, prohíbe la contratación de trabajadores en los Hospitales de la Red Pública Distrital, a través de las cooperativas de trabajo asociadas. Actualmente sólo el 17.4\% de los trabajadores de los hospitales de la red pública son de planta y el $82 \%$ están por contrato (fijo, prestación de servicios, temporal) (datos de la Secretaría de Salud 2011) hay en las modalidades de contratación del hospital, eso genera inequidad, los de planta tienen unas condiciones, los contratistas a través de cooperativas tienen otras condiciones, los de outsourcing tienen otras condiciones, de tal manera que no son iguales las condiciones para todos y, en últimas, las personas que son contratistas o cooperados en el hospital tienen condiciones desfavorables. (Médica)

(...) pues contento porque tengo un trabajo estable, aparentemente estable, pero si vemos en otro aspecto, como le puedo explicar, nosotros estamos muy, muy, muy mal por estar vinculados a una cooperativa, la cooperativa llámese como se llame, porque casi la mitad del salario se lo lleva la cooperativa. (Camillero)

Desde los discursos, se hace evidente un lenguaje ambiguo con respecto a los diferentes tipos de trabajador, ya que, por un lado, los funcionarios del área asistencial manifiestan la existencia de una diferencia entre los dos tipos de trabajadores - cooperados y de planta-, como se vio en los anteriores fragmentos, y, por otro lado, el personal de Recursos Humanos asegura que no existen diferencias.

No, no nosotros no tenemos distinciones entre planta y contrato; acá se sabe que somos mil y pico los que trabajamos y con esos mil y pico trabajamos; o sea nosotros hacemos el despliegue comunicacional para todo el mundo, hacemos el despliegue de beneficios para todo el mundo, sin distinción alguna. (Gerente de Talento Humano)

Esta ambigüedad refleja una contradicción que se pone en evidencia ante el aumento progresivo del indicador de rotación. En ese orden de ideas, se hace difícil fidelizar a los trabajadores debido a la coexistencia de estrategias de integración y alineación del personal a la organización y, al mismo tiempo, formas de contratación flexible. Esta fluidez y liquidez del trabajador corresponden a una nueva cultura laboral que se está configurando en el contexto del trabajo contemporáneo, caracterizado por el predominio de condiciones de trabajo flexibles e inseguros (Bauman, 2002; Sennett, 
2000). De esta manera, la cultura de la flexibilidad promovida por las nuevas formas de contratación, entran en contradicción con los esfuerzos que hacen las empresas por construir culturas fuertes, en las que los trabajadores estén alineados con la filosofía corporativa.

Porque la empresa no puede decir: yo voy a pagarle mejor para que usted no se me vaya, yo lo voy a "exclusivizar" a usted; porque el sistema presupuestal de estas empresas no permite hacer eso, (...) con los especialistas se han hecho esfuerzos grandes por mantenerlos, porque es otro personal que rota altísimo. (Coordinador de Calidad)

Legge (1995) señala que el nuevo discurso de gestión humana recurre a un tono afectivo -propio del proceso de gestión cultural- que, según esta autora, busca involucrar afectivamente al trabajador -alineación afectiva- de manera que este justifique y acepte las crudas decisiones que se toman en la organización; un discurso, que en medio de las condiciones de precariedad, busca perpetuar el vínculo del trabajador hacia su empresa, a pesar de que la empresa a través de las nuevas formas de contratación, no se vincule ni se comprometa con los trabajadores.

De otro lado, la existencia de diferentes categorías de trabajadores expresadas en condiciones desiguales, genera estados de insatisfacción que pueden afectar las relaciones interpersonales y el clima laboral.

Si usted, de hecho, dice: pero, yo por qué no tengo derecho a unas vacaciones remuneradas como las tienen los demás de planta; ellos se pueden ir quince días a descansar y yo no puedo, y si yo dejo de trabajar me lo dejan de pagar; obviamente, de ahí en adelante, así sea uno muy buena gente, llega el momento en que se siente en desventaja y le da rabia contra el mundo y se puede estrellar o con el compañero o con el paciente, o tiene que ser una persona con una personalidad a toda prueba para no sentir nada y eso hace que sea difícil. (Médica)
De esta manera, el nivel rotación y las dificultades en las relaciones interpersonales en este caso, muestran que las prácticas de gestión humana entre cuyas metas están la integración del trabajador a la empresa y la promoción de un buen clima laboral, entran en contradicción con el impacto que generan las nuevas condiciones laborales de los trabajadores, caracterizadas por el aumento de la inseguridad laboral. Como señala Legge (1995), la Gestión Humana a través de un discurso de integración busca encubrir esta contradicción.

\section{Impacto de la Cultura de Calidad}

En el proceso de construcción de una cultura de calidad, de acuerdo con la Gerente de Gestión Humana, se ha logrado, en gran medida, apropiación e identidad con la filosofía de calidad por parte de los trabajadores:

Hemos generado una cultura básica, no puedo decir que hemos generamos una cultura iufff!... avasalladora, no..., pero hemos generado la cultura básica de que la gente se sienta movida por la calidad, que si yo hago las cosas por este lado, me van a salir bien, que si yo me actualizo, mis procedimientos, las cosas me van a salir bien. (Gerente de Talento Humano)

De otro lado, según el siguiente fragmento, la evidencia del impacto de la Gestión de la Cultura de Calidad, en los comportamientos de los trabajadores, es la certificación ISO.

En conjunto, todo el trabajo en calidad tiene que haber impactado el comportamiento individual de todos, si no el hospital no habría podido conseguir la certificación ISO (...) si no hubiera cambiado la gente realmente no podríamos tener certificaciones. (Médica)

Pareciera ser que la certificación es la evidencia de que la calidad es una realidad vivida en la organización; no obstante, fenómenos, como el incremento de la inseguridad laboral, dan cuenta 
de un significado predominante de calidad que privilegia el ahorro de costos laborales, en detrimento del bienestar de los trabajadores.

Por otro lado, el impacto de la Cultura de la Calidad, no es el mismo en el personal asistencial que en el personal administrativo, ya que según los siguientes fragmentos, el lenguaje de calidad es ajeno a las actividades asistenciales. Este discurso se ajusta más a actividades administrativas que son más fácilmente traducibles en términos numéricos.

Las cosas relacionadas con todos esos procesos de calidad, de la norma, de control documental, de procedimientos escritos, de auditoría, creo que han permeado un poco más a la parte administrativa; en eso, creo que el desarrollo es mayor, es muchísimo más difícil hacia la parte asistencial, porque el idioma que se habla en los sistemas de gestión de calidad es muy difícil traducirlo a mi actividad diaria asistencial. (Médica)

El lenguaje de calidad no está involucrado dentro de nuestra actividad diaria con el paciente, y donde es muy difícil que los asistenciales lo asimilen, lo vivan de esa manera. (Médica)

Es así como se encuentran dificultades por parte del personal asistencial en la comprensión del lenguaje de calidad, el cual está formulado en términos administrativos y estadísticos, ajenos a sus prácticas cotidianas. Por el contrario, para los funcionarios del área asistencial, la calidad está más orientada hacia el tipo de relación que se construye con el paciente y el efecto de esta interacción en el bienestar de éste. Es en la interacción con el paciente, el escenario donde el personal asistencial puede comprender y valorar la realidad y magnitud de la problemática; sin embargo, la racionalización del tiempo de atención (García, 2007) no favorece este proceso y, por el contrario, pone en evidencia la importancia de la lógica económica que sostiene al hospital como empresa. En ese sentido el leguaje de calidad -indicadores, cifras, tiempos- resulta ajeno a la realidad experimentada por el personal asistencial.

La distancia que sienten los trabajadores asistenciales con respecto al discurso de calidad, se manifiesta, de otro lado, en la diferencia entre el personal administrativo y asistencial, debido a las distintas lógicas que estos grupos manejan.

Conciliar con los administrativos y asistenciales (...), sigue habiendo una brecha que nos separa. Los asistenciales piensan que los administrativos no comprenden nuestro quehacer; es muy bonito allá en el escritorio que es muy distinto a tener las manos dentro del paciente, eso hace que de pronto la relaciones tampoco se vean fáciles. (Médica)

Los cambios que se han dado en los hospitales y que responden a la lógica empresarial (Valdés, 2008), han llevado a que termine imponiéndose el lenguaje administrativo, al que los trabajadores asistenciales deben ajustarse para poder sobrevivir en el mercado laboral, a pesar de la dificultad que tienen para entenderlo, apropiarlo e incorporarlo.

De otro lado, al indagar sobre el impacto del proceso de calidad en el paciente, los trabajadores afirman que el nivel de satisfacción de los usuarios frente al servicio prestado es alto.

Pero a nivel de satisfacción, aquí se maneja un noventa por ciento de satisfacción, haciéndolo por una encuesta construida por nosotros mismos, la hemos ido mejorando; de aquí al año entrante, se piensa hacer únicamente con evaluador externo, entonces ya tenemos un gran pulso de lo que dice el usuario. (Coordinador de Calidad)

En la institución, la atención al paciente yo creo que sí ha mejorado, ehh... cuando yo llegué veía uno filas desde las cuatro de la mañana, allá la gente haciendo fila con el frío, los viejitos, hoy en día sí hay colas, pero menos, hay filas preferenciales, ehh... se ha trabajado muchísimo en el tema de humanización, que eso falta cantidades, pero se ha logrado la integración de algunas maneras de la comunidad a través de la asociación de usuarios (...), entonces la atención de los pacientes sí creo que se ha cambiado mucho, aunque haya muchísimo todavía por cambiar (...). (Enfermera) 
De acuerdo con el Coordinador de Calidad existen indicadores financieros y técnicos-asistenciales, dentro de los últimos se encuentran:

(...) indicadores de desempeño hospitalario que son las estancias de los pacientes, el promedio de días que dura un paciente acá, el giro de la cama, cuántas veces se ocupa una cama durante un mes, indicadores de utilización, cuántos laboratorios por egreso utilizó un paciente, cuántas imágenes diagnósticas por egreso utilizó un paciente, los indicadores relacionados con la infección hospitalaria y con el seguimiento de los eventos adversos, la satisfacción de los clientes, el estado de las acciones de mejora.

Se supone que los indicadores asistenciales permiten medir si se está cumpliendo con los niveles requeridos de atención para garantizar un servicio de salud de calidad, sin embargo, estos enfatizan en aspectos que tienen que ver con la racionalización de recursos -cuántos laboratorios por egreso, cuántas imágenes diagnósticas por egreso-, mostrando una lógica económica que a la vez sugiere como buena práctica la restricción en la prescripción (García, 2007).

Según los discursos, existe consenso en cuanto a la manera como perciben el impacto positivo de la Gestión de Calidad en los pacientes. No obstante, el siguiente fragmento ilustra cómo las categorías e indicadores de calidad pueden invisibilizar realidades como la siguiente:

Mire, yo ayer atendí a un paciente que llegó ahí, es un muchacho que está prácticamente solo en la vida, lo está apoyando una señora del lado de Puente Aranda, le tocaba hacerle electrocardiograma, le valía 98.800 pesos, no se lo pudo hacer porque no tenía ni un peso, ni está trabajando, tiene un soplo en el corazón, no está trabajando, estaba alejado de la familia, ¿qué tiene que hacer este muchacho? Dejarse morir, él no tiene Sisbén, es decir no tiene nada. (Enfermera)

En ese sentido los indicadores, como se señaló anteriormente, visibilizan ciertas realidades y a la vez invisibilizan otras, por ejemplo, el impacto de la racionalización (del tiempo en la atención, de recursos) en la prestación del servicio y en las condiciones laborales. Así, los indicadores de calidad vigentes, al no mostrar estas realidades, llevan a legitimar el funcionamiento del sistema de salud.

Se observa cómo este hospital se convierte en un hospital modelo, en tanto administra eficientemente los recursos financieros logrando la autosostenibilidad, y al mismo tiempo consigue mostrar un buen nivel de satisfacción de los usuarios. Sin embargo, estos indicadores y discursos han permitido legitimar un modelo de salud en donde, por ejemplo, la negación de exámenes a los usuarios, se convierte en una práctica común y al mismo tiempo invisible, en relación con los indicadores. Al respecto, según un informe de la Procuraduría General del la Nación (2008) acerca del derecho a la salud, el número de tutelas interpuestas por ciudadanos para que se les garantice el derecho a la salud, ante la negación del servicio, aumenta constantemente. De otro lado, un gran porcentaje de los casos de pacientes asesorados por Salud al Derecho $^{7}$, tanto del régimen subsidiado como del contributivo, han sufrido diversas formas de negación en la atención (Abadía-Barrero \& Oviedo Manrique, 2009).

Por último, la cultura de la evaluación inherente a los sistemas de calidad ha venido permeando a los trabajadores del hospital, quienes legitiman la supervisión y medición constante sobre sus acciones; de esta manera se evidencian cambios en las conductas y actitudes de las personas, producto de este seguimiento, como se señala en el siguiente fragmento.

La cultura de aceptar que se les mida de alguna manera por indicadores en el tema de gestión am-

7 La Asociación Colombiana para la Exigibilidad del Derecho a la Salud y Seguridad Social-Salud al Derecho- es una organización social que surge en el 2002, para contribuir a dar respuesta a las violaciones al derecho a la salud, por parte de instituciones del sistema. Su quehacer está orientado a "brindar apoyo y adelantar, con criterio humanista y solidario, toda clase de acciones y actividades orientadas a la defensa, promoción, construcción y realización de los derechos fundamentales a la seguridad social, a la salud pública y en general a todos los derechos humanos, económicos, sociales y culturales (Salud al Derecho, 2007 citado en Oviedo, Abadía \& Martínez., 2008). 
biental, se ha logrado; la clasificación de desechos, el tema de accidentes laborales, el tema de la atención segura a los pacientes con respecto a las infecciones adquiridas en el hospital, la gente se lava las manos más ahora, ehh... entonces si se ha logrado cambios actitudinales en las personas. (Médica)

Los años que lleva el hospital implementando el sistema de Gestión de Calidad y con esto, la cantidad de veces que los trabajadores se han expuesto a todo tipo de evaluaciones tanto internas como externas, ha llevado a naturalizar el tema de las evaluaciones. La evaluación es una práctica inherente al trabajo cotidiano y por eso, a pesar de que en el siguiente fragmento se afirma que la gente lo ve como un desgaste, existe todo un ritual de preparación previo a las auditorías.

La gente se ha acostumbrado, creo yo, son tantas veces y tantos que nos han venido a evaluar, que la auditorías del Icontec, de la Veeduría, que la que la Contraloría, mejor dicho, es que eso es pan de todos los días. La gente siente eso de alguna manera como desgastante, quitan mucho tiempo, a veces vienen personas que no están preparadas técnicamente, pero en general la gente está dispuesta, se prepara para las auditorias, entonces cuando vienen las auditorías la gente estudia y ahí nos ponen la 'copialina', estudiamos, (...) ahorita entramos en la cultura de que evidencias es tomar fotos, ahí está lo foto, no son mentiras. (Médica)

En relación con la evaluación del desempeño, el hospital intenta extender esta práctica no sólo a los trabajadores de planta, sino también a los cooperados, y se diseñan nuevas estrategias que buscan evaluar la productividad de los trabajadores, algo que podría ir en contra de la práctica asistencial.

Formalmente, en este momento las únicas personas que tienen una evaluación del desempeño real, son las personas del plan de carrera administrativa; ellos tienen un instrumento diseñado, pues, por el Servicio Civil; personalmente, pienso que es un instrumento completamente inadecuado, esa es mi percepción personal (...) en este momento se está empezando a implementar sistemas de evaluación individual para los cooperados, y están tratando de hacer evaluar la productividad personal a cada persona, eso está generando choque en esta institución, está generando un choque de trenes, porque evaluar la productividad individual en actividades asistenciales es un terreno complicado (...) entonces lo que se va afectar es el bolsillo. (Médica)

Esto pone de relieve la importancia que tienen las prácticas productivas -o que no generen pérdidas económicas- en la intervención sobre el paciente, y de la no productividad, responde cada trabajador de manera individual.

\section{Conclusiones}

Este trabajo ilustra cómo la Gestión Humana, a través de la utilización de tecnologías psicológicas, contribuye a la reproducción de un orden social y unos intereses particulares, haciendo evidente el carácter político de dichas tecnologías (Rose, 1998). La Gestión Cultural, en el marco de la gerencia de Recursos Humanos, se ha convertido en una tecnología social por excelencia, para la administración y gobierno de los grupos humanos insertos en las organizaciones, promoviendo un proceso de integración y alineación, en medio de un contexto donde se acentúa la flexibilización y precarización laboral (Legge, 1995). La intención de estudios como este es invitar a reflexionar acerca del papel que ha tenido la Psicología, y en particular la Psicología Organizacional, en el proceso de legitimación de las transformaciones en el contexto del trabajo contemporáneo. Particularmente, en este estudio se muestra cómo la dinámica organizacional no se puede entender ajena a los procesos sociales, económicos y políticos del contexto nacional e internacional, y cómo las organizaciones, en este caso los hospitales, responden al modelo de mercado dominante, en el que la salud se transforma en un bien de consumo y el trabajador en un recurso, manejado según las necesidades de la empresa. En este proceso de incorporación de las organizaciones al mercado, y en particular de los hospitales, se suscita una serie de contradicciones al 
entrar en conflicto los imperativos de rentabilidad, por un lado, y los intereses del trabajador por tener un trabajo digno y del ciudadano en general por tener garantizado su derecho a la salud. Algunas de estas contradicciones son ilustradas en este trabajo.

El trabajador, en el marco del programa de Gestión Cultural, es concebido como un elemento fundamental en el proceso de consolidación de la cultura corporativa. Los esfuerzos por integrar al individuo reflejan una concepción de un trabajador esencial para el logro de los objetivos corporativos, a través de la incorporación que este hace de la filosofía organizacional y la materialización del discurso, a través de sus acciones y prácticas. Pero, por otro lado, y paradójicamente, las nuevas formas de contratación flexible muestran una concepción del trabajador como un recurso o un costo que debe ser reducido, para asegurar la sostenibilidad económica (Legge, 1995). En ese sentido, como lo señala la autora, el modelo de Gestión Humana entraña una concepción ambigua del trabajador: como eje fundamental en el proceso de producción -en el caso del hospital, la prestación del servicio de salud- y, por otro lado, como un costo que debe ser minimizado, o un recurso que se mueve de acuerdo con las necesidades de la organización.

Este significado ambiguo del trabajador se refleja en la configuración de un trabajador más volátil. Así, la inversión que hace la organización en el trabajador a través de procesos de formación, lleva, paradójicamente, a transformarlo en sujeto atractivo en el mercado laboral, siendo su volatilidad, un costo para la organización. De esta mane$\mathrm{ra}$, las estrategias implementadas para integrar al trabajador a la cultura corporativa, se convierten en una oportunidad para este en la búsqueda de otras opciones laborales.

En relación con el lenguaje de calidad, se observa cómo los indicadores que se utilizan para medir el trabajo, reflejan una lógica administrativa que enfatiza la racionalización de costos, tapando realidades como por ejemplo la del impacto de esta racionalización de recursos en la salud del paciente (Abadía-Barrero \& Oviedo Manrique, 2009), o en las condiciones laborales de los trabajadores
(García, 2008). Sin embargo, toda esta lógica económica que sustenta el modelo de calidad, se ve disimulada por el lenguaje técnico (cifras), desde el que se busca demostrar objetividad y neutralidad.

A partir de los indicadores se busca traducir las acciones en evidencias objetivas; una objetividad que muestra las realidades que se quieren evidenciar. Como señala Rose (1997) “(...) [Estas medidas], al poner el énfasis en la aparente objetividad y neutralidad de los números, refuerzan la pretensión de esas entidades, de que actúan de acuerdo con un programa apolítico" (p. 36). No obstante, lejos de ser apolíticas, estas medidas sustentan un modelo de salud apoyado en una lógica de mercado.

\section{Referencias}

Abadía-Barrero, C. E., Cortés. G., Fino, D., García, C. M., Oviedo, D., Pinilla, M. Y. et al. (2008). Perspectivas inter-situadas sobre el capitalismo en la salud: desde Colombia y sobre Colombia. Palimpsesto, 6, 177-190.

Abadía-Barrero, C. E. \& Oviedo Manrique, D. G. (2009). Bureaucratic itineraries in Colombia. A theoretical and methodological tool to assess managed-care health care systems. Social Science \& Medicine, 68 (6), 1153-1160.

Bauman, Z. (2002). Modernidad líquida. Buenos Aires: Fondo de Cultura Económica.

Bonilla, E. \& Rodríguez, P. (1997). Más allá del dilema de los métodos: la investigación en ciencias sociales. Bogotá: Editorial Universidad de los Andes.

Castells, M. (1998). La era de la información: Economía, sociedad y cultura. La sociedad red (Vol. 1). Madrid: Alianza Editorial.

Colombia, Ministerio de la Protección Social. (2008). Manual de calidad. Disponible en http://www.minproteccionsocial.gov.co/VBeContent/library/documents/DocNewsNo17571DocumentNo8055. PDF

Colombia, Procuraduría General de la Nación. (2008). El Derecho a la Salud en perspectiva de Derechos Humanos y el Sistema de Inspección, Vigilancia y Control del Estado Colombiano en Materia de Quejas en Salud. Bogotá: Autor. 
De la Garza, E. (2002). Cultura y subjetividad: el caso del Sindicato de Telefonistas de la República Mexicana. Espacio Abierto, 11 (1), 65-94.

Escobar, A. (2005). Trabajo, desocupación y configuración de la subjetividad laboral en la región del Biobío, Chile. Trabajo y Sociedad. Indagaciones sobre el empleo, la cultura y las prácticas políticas en sociedades segmentadas, 6 (7), 1-19.

Kerguelén, C. (2003). Calidad en Salud iQuées? Bogotá: Editorial Centro de Gestión Hospitalaria.

García, C. (2006). Una aproximación al concepto de cultura organizacional. Universitas Psicológica, 5 (1), 163-174.

García, C. (2007). El hospital como empresa: nuevas prácticas nuevos trabajadores. Universitas Psychologica, 6 (1), 143-154.

García, C. (2008). Subjetividades laborales: impacto de la reforma de la salud en trabajadores de tres hospitales públicos de Bogotá. Revista Pretil, 17, 28-44.

García, C. (2009). La cultura organizacional como una tecnología de control gerencial. En Psicología del Trabajo y de las Organizaciones. Reflexiones y experiencias de investigación (pp. 275-295). Bogotá: Editorial Universidad Santo Tomás.

García, C. \& Carvajal, L. M. (2007). Tecnologías empresariales del yo: la construcción de sujetos laborales en el contexto del trabajo inmaterial. Universitas Psicológica, 6 (1), 49-58.

Gorz, A. (1997). Miserias del presente, riqueza de lo posible. Barcelona: Paidós Ibérica.

Legge, K. (1995). Human resource management: Rhetorics and realities. Basingstoke, UK: MacMillan Business.
Luengas, S. (2003). El Sistema de Habilitación: algo más que los nuevos requisitos esenciales. Revista Vía Salud, 22, 11-33.

Mumby, K. (1997). The problem of hegemony: Rereading Gramsci for organizacional communication studies. Western Journal of Communication, 61 (4), 343-375.

Oviedo, D., Abadía, C. \& Martínez, A. (2008). Movilización social por la defensa del derecho a la salud en Bogotá: esfuerzos locales y conexiones globales. Revista Pretil, 17, 46-63.

Peters, T. \& Waterman, R. (1984). En búsqueda de la excelencia. Bogotá: Editorial Norma.

Rose, N. (1997). El Gobierno en las democracias liberales "avanzadas": del liberalismo al neoliberalismo. Cuadernos de Critica de la Cultura, 29, 25-40.

Rose, N. (1998). Inventing our selves: Psychology, power and personhood. Cambridge, UK: Cambridge University Press.

Rose, N. (1999). Governing the soul: The shaping of the private self. London: Free Association Books.

Schein, E. (1985). Organizational culture and leadership. San Francisco: Jossey-Bass.

Sennett, S. (2000). La corrosión del carácter. Barcelona: Anagrama.

Urrea, F. (1999). Globalización, subcontratación y desregulación laboral. En L. G. Arango \& C. M. López (Comps.), Globalización, apertura económica y relaciones industriales en América Latina (pp. 50-92). Bogotá: Centro de Estudios Sociales, Universidad Nacional de Colombia.

Valdés, E. (2008). La transformación de la gestión hospitalaria en el Distrito Capital. Revista Pretil, 17, 64-84. 
